\title{
Computability Theory and Differential Geometry
}

\author{
Robert I. Soare*
}

December 1, 2003

\section{Contents}

1 Introduction 3

2 Poincaré and Hilbert $\quad 4$

3 Undecidable problems $\quad 6$

3.1 The word problem and triviality problem . . . . . . 6

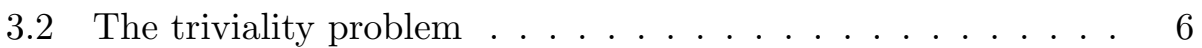

3.3 The homeomorphism problem . . . . . . . . . . 7

3.4 Other undecidability results in topology . . . . . . . 8

3.5 Novikov's Theorem on diffeomorphisms . . . . . . . . . 9

4 Some basic differential topology 9

4.1 Smooth manifolds and smooth maps . . . . . . . . . . 10

4.2 Derivatives on Manifolds . . . . . . . . . . . . . . . . 11

4.3 Tangent spaces . . . . . . . . . . . . . . . . . . 12

4.4 Immersions and hypersurfaces . . . . . . . . . . . . . . 12

4.5 Fundamental groups and homology groups . . . . . . . . . 13

4.6 The Generalized Poincaré Conjecture . . . . . . . . . . . . . . 14

4.7 Connected sum . . . . . . . . . . . . . . . . 15

*The author was supported by National Science Foundation Grants DMS 98-02619 and 00-99556. He presented some of these results as a plenary speaker at Centennial Meeting of the Association for Symbolic Logic in Urbana, Illinois, in June, 2000. The AMS Classification Code is 03D25. The author is grateful for helpful suggestions, corrections, and conversations with geometers and topologists: Benson Farb, Charles Livingston, John Lott, Alex Nabutovsky, Jan Reimann, and others. The author is particularly grateful to Shmuel Weinberger for many hours of discussion about these results and the connections between differential geometry and computability. 
5 Riemannian geometry $\quad 15$

5.1 Riemannian metrics . . . . . . . . . . . . 16

5.2 Isometries . . . . . . . . . . . . . . . . 16

5.3 Immersed manifolds . . . . . . . . . . . . . . . . 17

5.4 The length of paths in $M \ldots \ldots \ldots \ldots$

6 Unsolvable fundamental groups and geodesics 18

6.1 The covariant derivative and geodesics . . . . . . . . . 18

6.2 The Fundamental Group and Geodesics . . . . . . . . . . 18

6.3 Curvature . . . . . . . . . . . . . . . . . . . 19

7 The Geometric Space Riem/Diff 19

$7.1 \operatorname{Met}(M) \ldots \ldots \ldots \ldots \ldots \ldots \ldots$

7.2 The Gromov-Hausdorff metric . . . . . . . . . . . 20

7.3 A path metric on $A \ell(M) \ldots \ldots \ldots \ldots . \ldots . \ldots 21$

7.4 Local minima on $\operatorname{Met}(M) \ldots \ldots \ldots . \ldots . \ldots 22$

7.5 Sublevel sets . . . . . . . . . . . . . . . 22

8 Computability results for differential geometry 23

8.1 Standard definitions . . . . . . . . . . . . . 23

8.2 Dominating the settling time . . . . . . . . . . 24

8.3 A dominating sequence . . . . . . . . . . . . 25

9 The Nabutovsky Weinberger (NW) Results 26

9.1 C.E. degrees and local minima . . . . . . . . . . 26

9.2 The idea of the proof . . . . . . . . . . . . . 28

9.3 How deep is deep? . . . . . . . . . . . . . . . . . . . . 29

9.4 Using the settling function . . . . . . . . . . . . 30

9.5 The simplicial norm and lower bounds on volume . . . . . . . 31

\begin{abstract}
Let $M$ be a smooth, compact manifold of dimension $n \geq 5$ and sectional curvature $|K| \leq 1$. Let $\operatorname{Met}(M)=\operatorname{Riem}(M) / \operatorname{Diff}(M)$ be the space of Riemannian metrics on $M$ modulo isometries. Nabutovsky and Weinberger studied the connected components of sublevel sets (and local minima) for certain functions on $\operatorname{Met}(M)$ such as the diameter. They showed that for every Turing machine $T_{e}, e \in \omega$, there is a sequence (uniformly effective in $e$ ) of homology $n$-spheres $\left\{P_{k}^{e}\right\}_{k \in \omega}$ which are also hypersurfaces, such that $P_{k}^{e}$ is diffeomorphic to the standard $n$-sphere $S^{n}$ (denoted $P_{k}^{e} \approx_{\text {diff }} S^{n}$ ) iff $T_{e}$ halts on input $k$, and in this case the connected sum $N_{k}^{e}=M \# P_{k}^{e} \approx_{\text {diff }} M$, so $N_{k}^{e} \in$ $\operatorname{Met}(M)$, and $N_{k}^{e}$ is associated with a local minimum of the diameter
\end{abstract}


function on $\operatorname{Met}(M)$ whose depth is roughly equal to the settling time $\sigma_{e}(k)$ of $T_{e}$ on inputs $y<k$.

At their request Soare constructed a particular infinite sequence $\left\{A_{i}\right\}_{\in \omega}$ of c.e. sets so that for all $i$ the settling time of the associated Turing machine for $A_{i}$ dominates that for $A_{i+1}$, even when the latter is composed with an arbitrary computable function. From this, Nabutovsky and Weinberger showed that the basins exhibit a "fractal" like behavior with extremely big basins, and very much smaller basins coming off them, and so on. This reveals what Nabutovsky and Weinberger describe in their paper on fractals as "the astonishing richness of the space of Riemannian metrics on a smooth manifold, up to reparametrization." From the point of view of logic and computability, the Nabutovsky-Weinberger results are especially interesting because: (1) they use c.e. sets to prove structural complexity of the geometry and topology, not merely undecidability results as in the word problem for groups, Hilbert's Tenth Problem, or most other applications; (2) they use nontrivial information about c.e. sets, the Soare sequence $\left\{A_{i}\right\}_{i \in \omega}$ above, not merely Godel's c.e. noncomputable set $\mathrm{K}$ of the 1930's; and (3) without using computability theory there is no known proof that local minima exist even for simple manifolds like the torus $T^{5}$ (see $\S 9.5$ ).

\section{Introduction}

The main point of this paper is to explain the differential geometry results in $\S 9$ which appear in [NW1] and [NW2] and which are the most recent, having undergone several revisions. The computability results used in them are presented in $\S 8$, and will be proved in full in Csima and Soare [ta]. The geometry results depend upon and extend a number of undecidability results in algebra and topology which we review in $\S 3$, especially the AdjanRabin result about the unsolvability of the triviality problem for groups, and Novikov's theorem on the unsolvability of the diffeomorphism problem.

The differential geometry results require a large background in topology and geometry. To make the presentation as self-contained as possible we devote several sections to a review and intuitive description of these mathematical elements most needed. In $\S 4$ we discuss smooth manifolds, tangent spaces, fundamental groups, the Poincaré Conjecture, and the connected sum. In $\S 5$ we discuss Riemannian geometry, Riemannian metrics (as studied by Nabutovsky and Weinberger), isometries, and the metric space metric derived from the Riemannian metric. In $\S 6$ we look at a preliminary result to Nabutovsky and Weinberger's work, namely Gromov's theorem that a smooth manifold whose fundamental group has an unsolvable word problem 
has infinitely many contractible, closed geodesics. With this background, we introduce in $\S 7$ the space $\operatorname{Met}(M)=\operatorname{Riem}(M) / \operatorname{Diff}(M)$ of Riemannian metrics modulo diffeomorphisms upon which Nabutovsky and Weinberger obtain their results. $\operatorname{Met}(M)$ is a space whose points are metrics but it itself has a metric, the Gromov-Hausdorff metric and the path metric derived from it which is used in the Nabutovsky Weinberger results in $\S 9$. Also defined here are the notion of local minimum and sublevel set as used in $\S 9$.

\section{Poincaré and Hilbert}

A hundred years ago two men dominated the mathematical landscape. The first man, Henri Poincaré, working in Paris, used only constructive proofs, did not accept the completed infinite, and was not sympathetic to logic. He was keenly interested in the combinatorial study of connectivity of spaces, and with the properties of a system which endure when metric distortion occurs, namely the subject of topology. Poincaré [1895] introduced a new way to associate with a topological space $X$ a group which measured its complexity under connectivity. That group bore his name, the Poincaré group, and is now also known as the fundamental group, or the first homotopy group, $\pi_{1}(X)$, defined in $\S 4.5$. This opened the way for the modern study of algebraic topology and homotopy theory.

The second man, David Hilbert, working in Göttingen, Germany, believed in nonconstructive proofs and indeed had used a proof by contradiction in his famous solution of Gordan's Problem about solutions to a system of invariant forms. He believed in the completed infinite, but wanted to use logic to achieve consistency and avoid the paradoxes of set theory. He was also keenly interested in geometry. In 1898 he wrote Grundlagen der Geometrie, in which he reduced the consistency of Euclidean Geometry to the consistency of the arithmetic of the reals $\mathbb{R}$.

In Hilbert's famous address to the International Congress of Mathematicians in 1900, his Second Problem was to prove the consistency of arithmetic of $\mathbb{R}$. He soon realized that a more basic problem was to prove the consistency of $\mathbb{N}=(N,+, \times)$, which he announced in 1904 as an open problem. Hilbert's Tenth Problem at the 1900 ICM meeting was to give an algorithm to solve Diophantine equations. From 1900 until 1930 two of the main themes in Hilbert's work were proving the consistency and the decidability of various mathematical problems, chief among which was the Entscheidungsproblem, the decision problem for first order logic itself.

Hilbert had absolute faith that these decision procedures existed, and 
he believed: "There is the problem. Seek its solution. You can find it by pure reason." At Hilbert's retirement address in 1930 he said, "Wir müssen wissen. Wir werden wissen." We must know. We shall know. Ironically, only a year later in 1931 Gödel announced his famous Incompleteness Theorem that an effective axiomatization of even so simple a system as $\mathbb{N}=(N,+, \times)$ is either incomplete or inconsistent.

The intense interest by so prominent a mathematician as Hilbert in decidability and consistency led to a study of possible definitions for computable functions. Gödel [1931] had extensively used the primitive recursive functions, but only in 1934 defined the general recursive functions. After seeing the Turing computable functions in Turing [1936], Gödel emphatically agreed that Turing had finally found the right definition. This was shown to be extensionally the same as Gödel's own. (See Davis [1965.])

With a precise definition of computable function, mathematicians showed that many famous mathematical problems were unsolvable, as we examine in the next section. One of the key tools was the use of computably enumerable (c.e.) sets, those which are the range of a computable function. In the Nabutovsky-Weinberger (NW) papers c.e. sets are used not only for undecidability as in the past, but now for the first time to measure geometric complexity, such as the depth of basins. Nabutovsky and Weinberger use the idea of Novikov in $\S 3.5$ that it is an unsolvable problem whether a given manifold (homology sphere) is diffeomorphic to the standard $n$-sphere $S^{n}, n \geq 5$, which is defined in Definition 4.1. The NW results use c.e. sets $A$ not only as a switch to turn on or off a diffeomorphism, as in Novikov, but remarkably, they relate the settling time of the Turing machine on an argument $k$ to the depth of the corresponding basin and to the distribution of basins.

These results one hundred years after Poincaré [1895] and Hilbert's 1900 ICM address are not at all what they expected. Poincaré would probably have been skeptical of using logic in geometry, and would never have understood the vast distances in Met(M) obtained by NW using computable functions and c.e. sets. Hilbert would never have understood noncomputable functions, c.e. sets, or their use to demonstrate geometric complexity since he yearned for consistency and decidability. Nevertheless, Poincaré gave us the combinatorial study of connectivity properties, and Hilbert's program led to the definition of computable functions and their applications using c.e. sets. Both loved and advanced geometry or topology. Both were highly original thinkers who moved beyond the boundaries of the conventional thinking of their day. The present NW results, weaving fundamental groups and c.e. sets to demonstrate vast depths, are a beautiful fruition and combination of 
the consequences of these two fundamental mathematical traditions.

\section{$3 \quad$ Undecidable problems}

Turing [1936] gave a negative solution to the Entscheidungsproblem and proved the existence of unsolvable problems in mathematics. Building on work of Davis, Putnam, and Julia Robinson, Matijasevich proved in 1970 that Hilbert's 10th Problem on Diophantine equations is computably unsolvable. He showed that c.e. sets $A$ can be represented by Diophantine predicates and used the unsolvability of the halting problem, i.e., of the membership problem "is $x \in A$ ?" See Davis [1973]. More relevant to the present paper is the work growing out of the word problem. (In the later sections $\S 4-\S 7$ we review the definitions and results from geometry and topology used in this paper.)

\subsection{The word problem and triviality problem}

The unsolvability results about topological decision problems which have been obtained so far have been derived from the unsolvability of the word problem (see Boone [1955], Novikov [1955]), or the triviality problem (see Adjan [1955], and Rabin [1958]). These unsolvable group theoretic problems also play a key role in the present Nabutovsky-Weinberger results.

A group $G$ is finitely presented (f.p.) if there are a finite number of generators $S=\left\{g_{1}, \ldots, g_{k}\right\}$ and finitely many relations $Q=\left\{R_{1}, \ldots R_{n}\right\}$ which are words (finite strings) on $g_{i}^{ \pm 1}$. The word problem for $G$ is to decide for an arbitrary word $x$ on the symbols in $S$, whether $x$ can be reduced to the empty word via finitely many applications of the relations in $Q$. We say that sets $A$ and $B$ have the same Turing degree denoted by $A \equiv_{T} B$ if $A$ is computable in $B$, written $A \leq_{\mathrm{T}} B$, and $B \leq_{\mathrm{T}} A$.

Theorem 3.1 (Boone (1954), Novikov (1955)) The word problem is unsolvable, and indeed can have the same Turing degree as any c.e. set (Boone [1966]).

A good proof of the unsolvability of the word problem can be found in Rotman [1995], together with related material.

\subsection{The triviality problem}

The isomorphism problem is to decide for two finitely presented (f.p.) groups $G$ and $H$ whether they are isomorphic, and the triviality problem is to decide 
whether $G$ is the trivial group $\langle e\rangle$ (i.e., consisting only of the identity $e$ ). A c.e. set $C$ is $m$-complete if for every c.e. set $A$ there is a computable function $f$ such that $n \in A$ iff $f(n) \in C$, because $C$ codes at least as much information as any such $A$. (See Soare $[1987$, p. 41] for more information on $r$-completeness for various reducibilities $r$.)

\section{Theorem 3.2 (Adjan (1958b), Rabin (1958) Triviality Theorem)}

(i) The isomorphism problem is unsolvable, and the triviality problem is unsolvable.

(ii) Indeed, the triviality problem is $m$-complete for c.e. sets in the following sense. For any c.e. set $A$ there is a computable sequence of finitely presented groups $\left\{G_{k}\right\}_{k \in \omega}$ such that:

$$
k \in A \Longleftrightarrow G_{k} \cong\langle e\rangle .
$$

Property (ii) plays a key role in the NW results below and in the following earlier results on which they are based.

\subsection{The homeomorphism problem}

Lemma 3.3 Given a presentation $\mathbf{P}$ of a f.p. group $G$ one constructs a 2-dimensional complex $K^{2}(\mathbf{P})$ which corresponds to $\mathbf{P}$ whose fundamental group $\pi_{1}\left(K^{2}(\mathbf{P})\right)$ is isomorphic to $G$. (The fundamental group is defined §4.5.)

Proof (Sketch). (See Haken [1973, p. 431] for details.) Choose a point $O$ for the only vertex of $K^{2}(\mathbf{P})$. Corresponding to each generator $g_{i}$ in $\mathbf{P}$ choose a 1-dimensional cell $G_{i}$ of $K^{2}(\mathbf{P})$ which is an arc that originates and terminates at $O$. Except for their endpoints, the $G_{i}$ are chosen pairwise disjoint. Thus, $\bigcup_{i=1}^{k} G_{i}$ is a wedge of $k$ loops with fundamental group the free group of rank $k$. For each relation $R_{j}$ choose a 2-dimensional cell $H_{j}$ of $K^{2}(\mathbf{P})$ which is a disk whose boundary $\partial H_{j}$ is identified to a closed curve in $\bigcup_{1=1}^{k} G_{i}$ which runs through $G_{i}$ in the same order that $G_{i}$ occurs in $R_{j}$ (going in the same direction as $G_{i}$ for a positive occurrence of $g_{i}$ in $R_{j}$ and in the opposite direction for a negative occurrence of $g_{i}^{-1}$ in $R_{j}$ ).

The unsolvability of triviality problem Theorem 3.2 immediately implies the unsolvability of the simply connectedness (i.e., triviality of the fundamental group) for 2-complexes, and thus of related problems for 2-complexes,

In order to construct an $n$-dimensional manifold $M^{n}(\mathbf{P})$ with fundamental group $M^{n}(\mathbf{P}) \cong G$ corresponding to a group presentation $\mathbf{P}$, where $G$ 
is the group presented by $\mathbf{P}$, we may extend the 2-complex $K^{2}(\mathbf{P})$ into the Euclidean space $\mathbb{R}^{(n+1)}$, provided that $n \geq 4$. Choose a simplicial, regular neighborhood $N$ of $K^{2}(\mathbf{P})$ in $\mathbb{R}^{(n+1)}$. The fundamental group of $N$, but also the fundamental group of its boundary, is isomorphic to $G$.

From this we can conclude (Haken [1973, p. 432]) the unsolvability of the triviality problem, word problem, conjugacy problem, isomorphism problem of the fundamental groups for $n$-manifolds with $n \geq 4$. Markov made the remarkable discovery that we can also conclude the unsolvability of the homeomorphism problem for $n$-manifolds with $n \geq 4$. (See Haken [1973, p. 432].)

Theorem 3.4 (Markov, (1958)) For each dimension $n \geq 4$ the homeomorphism problem for $n$-manifolds is unsolvable.

Markov studied certain simple Tietze transformations, called Markov operations of a presentation $\mathbf{P}$ to another group presentation $\mathbf{P}^{\prime}$. He showed that if $\mathbf{P}^{\prime}$ is obtained from $\mathbf{P}$ by a Markov operation, then $M^{n}(\mathbf{P})$ is homeomorphic to $\mathbf{P}^{\prime}$. From this he showed that a solution of the homeomorphism problem for $n$-manifolds ( $n \geq 4$ ) would imply a solution of the triviality problem of group theory. (See Haken [1973, p. 432-433], and Boone, Haken, and Poenaru [1968].)

\subsection{Other undecidability results in topology}

Boone, Haken, and Poenaru [1968] extend this method to prove many stronger results such as the following. For this subsection only let $\approx_{1}, \approx_{2}$, $\approx_{3}$, and $\approx_{4}$ denote diffeomorphic, homeomorphic, combinatorially equivalent, homotopy equivalent respectively.

Theorem 3.5 (Boone, Haken, and Poenaru, (1968, Theorem 1)) For each dimension $\geq 4$ and for each computably enumerable degree $\mathbf{d}$ there is a computable class $\mathcal{C}(n, D)$ of finite presentations of $n$-manifolds, endowed with a differential and a compatible combinatorial structure, such that for each $1 \leq i \leq 4$ :

(i) the decision problem

$$
\left\{\left(\mathcal{M}_{1}, \mathcal{M}_{2}\right): \mathcal{M}_{1}, \mathcal{M}_{2} \in \mathcal{C}(n, d) \& \mathcal{M}_{1} \approx_{i} \mathcal{M}_{i}\right\}
$$

is unsolvable.

(ii) The decision problem

$$
\{\mathcal{M}: \mathcal{M} \in \mathcal{C}(n, D) \& M(\mathcal{M}) \text { is simply connected }\}
$$


has degree $\mathbf{d}$ where $M(\mathcal{M})$ is the manifold with presentation $\mathcal{M}$.

\subsection{Novikov's Theorem on diffeomorphisms}

Novikov improved Markov's Theorem 3.4 from "homeomorphic" to "diffeomorphic." A very good proof can be found in the Appendix of Nabutovsky [1995a, p. 86]. In addition, Markov proved only that there is no algorithm to decide for a pair of 4-manifolds whether or not they are homeomorphic, while Novikov proved that for any single 5 -manifold $M$ one cannot tell which manifolds $N$ are diffeomorphic to $M$.

Definition 3.6 Let $M \approx_{\text {diff }} N$ denote that manifolds $M$ and $N$ are diffeomorphic.

Theorem 3.7 (S. P. Novikov) For each dimension $n \geq 5$, and for each $n$-manifold $M$, it is unsolvable for an $n$-manifold $P$ whether $M \approx_{\operatorname{diff}} P$. (In particular, it is unsolvable whether $P \approx_{\operatorname{diff}} S^{n}$.)

Indeed, one can show for $n \geq 5$ that the diffeomorphism problem is $m$-complete in the sense that for every c.e. set $A$ there is an effective sequence of $n$-manifolds $\left\{P_{k}\right\}_{k \in \omega}$, such that

$$
k \in A \Longleftrightarrow P_{k} \approx_{\text {diff }} S^{n},
$$

where $S^{n}$ is the sphere in standard position, defined in $(2)$, and $\approx_{\text {diff }}$ denotes diffeomorphic. Very roughly, the idea is this. Construct each $P_{k}$ to be a homology sphere (i.e., to have the same homology groups as $S^{n}$ ), so that the homology of $P_{k}$ is not an obstacle to being diffeomorphic to $S^{n}$. Using the idea of the triviality problem above, construct a sequence of groups $\left\{G_{k}\right\}_{k \in \omega}$ such that $k \in A$ iff $G_{k}$ is trivial, and arrange that the fundamental group of $P_{k}$ is $G_{k}$. Further arrange that $P_{k} \approx S^{n}$ iff $G_{k}$ is trivial. If $k \notin A$ then the fundamental group is not trivial so $P_{k} \not S^{n}$. If $k \in A$ then the fundamental group is trivial and $P_{k} \approx S^{n}$. (See the Nabutovsky Weinberger extension of this idea in $\S 9.2$.)

\section{Some basic differential topology}

The Nabutovsky-Weinberger results are about local minima of the distance function for metrics on $\operatorname{Riem}(M)$, the space of Riemannian metrics of a certain manifold $M$. There are at least four different metrics we need to 
consider, Riemannian metrics on $M$, metric space metrics, the GromovHausdorff $(\mathrm{G}-\mathrm{H})$ metric on $\operatorname{Riem}(M)$, and the path metric defined from the G-H metric. To explain these carefully we need to review some basic differential and Riemannian geometry, and some algebraic topology for concepts like the fundamental group and homology spheres. Good introductory references for differential topology are Guillemin-Pollack [1974], and Milnor [1965]; for algebraic topology, [Massey, 1967]; and for Riemannian geometry, Petersen [1998], Cheval [1993], and Do Carmo [1992].

\subsection{Smooth manifolds and smooth maps}

Good references for this section are Milnor [1965] and Guillemin-Pollack [1974]. We mostly use Milnor's notation. Let $\mathbb{R}^{n}$ denote $n$-dimensional Euclidean space. A point $x \in \mathbb{R}^{n}$ is a $n$-tuple $\left(x_{1}, x_{2}, \ldots, x_{n}\right)$ of real numbers. A mapping $f$ of an open set $U \subset \mathbb{R}^{n}$ into $\mathbb{R}^{m}$ is smooth if it has continuous partial derivatives of all orders, i.e., is $C^{\infty}$. A mapping $f: X \rightarrow \mathbb{R}^{m}$ defined on an arbitrary subset $X \subseteq \mathbb{R}^{m}$ is smooth if it can be locally extended to a smooth map on open sets.

A smooth map $f: X \rightarrow Y$ of subsets of two Euclidean spaces is a diffeomorphism if it is one to one and onto, and if the inverse map $f^{-1}: Y \rightarrow X$ is also smooth. (In particular, $f$ is a homeomorphism from $X$ to $Y$.) A manifold $M$ is a $k$-dimensional manifold if it is a subset of $\mathbb{R}^{n}$ and is locally diffeomorphic to $\mathbb{R}^{k}$.

A diffeomorphism $\varphi: U \rightarrow V$ is called a parametrization of the neighborhood $V$, and the inverse diffeomorphism $\varphi^{-1}: V \rightarrow U$ is called a coordinate system of $V$. The subject of differential topology is the study of those properties of a set $X \subseteq R^{k}$ which are invariant under diffeomorphisms.

For example, the unit sphere

$$
S^{2}=\left\{(x, y, z): x^{2}+y^{2}+z^{2}=1\right\}
$$

is a smooth manifold of dimension 2. The diffeomorphism

$$
(x, y) \mapsto\left(x, y, \sqrt{1-x^{2}-y^{2}}\right)
$$

for $x^{2}+y^{2}<1$ parametrizes the region $z>0$ of $S^{2}$. By rearranging the roles of the variables and the regions $x>0, y>0, x<0, y<0$, and $z<0$ one obtains parametrizations for the latter regions. These cover $S^{2}$ which is therefore a smooth manifold. 
Definition 4.1 The sphere, sometimes called the unit sphere in standard position, $S^{n} \subset \mathbb{R}^{n+1}$, is a smooth manifold of dimension $n$ where

$$
S^{n}=\left\{\left(x_{1}, \ldots, x_{n+1}\right): \sum_{i=1}^{n+1} x_{i}^{2}=1\right\} .
$$

\subsection{Derivatives on Manifolds}

We define the derivative similarly as in a freshman calculus case, and then extend to several variables as in Spivak [1965]. Fix a smooth map $f$ from an open set in $\mathbb{R}^{n}$ into $\mathbb{R}^{m}$ and any point $x$ in the domain of $f$. For any vector $h \in \mathbb{R}^{n}$, define the derivative of $f$ in the direction $h$ taken at $x$ to be the usual limit

$$
d f_{x}(h)=\lim _{t \rightarrow 0} \frac{f(x+t h)-f(x)}{t}
$$

Therefore, for fixed $x$ we define a mapping $d f_{x}: \mathbb{R}^{n} \rightarrow \mathbb{R}^{m}$ by taking the vector $h \in \mathbb{R}^{n}$ to the directional derivative $d f_{x}(h)$. This is called the directional derivative of $f$ at $x$. In multivariate calculus courses one proves that the derivative mapping $d f: \mathbb{R}^{n} \rightarrow \mathbb{R}^{m}$ is linear (see Spivak [1965]) and hence may be represented by a matrix in terms of the standard basis. This matrix is the Jacobian matrix of $f$ at $x$ :

$$
\left(\begin{array}{lcr}
\frac{\partial f_{1}}{\partial x_{1}}(x) & \ldots & \frac{\partial f_{1}}{\partial x_{n}}(x) \\
\vdots & \vdots & \vdots \\
\frac{\partial f_{m}}{\partial x_{1}}(x) & \ldots & \frac{\partial f_{m}}{\partial x_{n}}(x)
\end{array}\right)
$$

The derivative operation obeys the next two fundamental properties.

Chain Rule. If $f: U \mapsto V$ and $g: V \mapsto W$ are smooth maps, with $f(x)=y$, then

$$
d(g \circ f)_{x}=d g_{y} \circ d f_{x} .
$$

Namely, to every commutative triangle of smooth maps between open sets $\subseteq \mathbb{R}^{n}$, there corresponds a commutative triangle of linear maps on $\mathbb{R}^{n}$. 
Identity. If $\mathrm{I}$ is the identity map on the open set $U \subset \mathbb{R}^{n}$, then $d I_{x}$ is the identity map of $\mathbb{R}^{n}$.

The close connection between diffeomorphisms and linear maps is brought into even sharper focus by the next remarkable theorem.

Theorem 4.2 (Inverse Function Theorem) Suppose that $f: X \mapsto Y$ is a smooth map with derivative $d f_{x}$ at the point $x$. If the linear map $d f_{x}$ is an isomorphism, then $f$ is a local diffeomorphism at $x$.

Note that $d f_{x}$ is an isomorphism iff the corresponding matrix has nonzero determinant, so that $d f_{x}$ in nonsingular. (For a proof of the Inverse Function Theorem see Dieudonné [1960, p. 268] or Spivak [1965].)

\subsection{Tangent spaces}

This connection between diffeomorphisms and linear maps, crucial for Riemannian metrics, is made even stronger by introducing the tangent space $T M_{x}$ of $M$ at a point $x \in M$. Choose a parametrization

$$
g: U \mapsto M \subseteq \mathbb{R}^{m}
$$

of a neighborhood $g(U)$ of $x \in M$ with $g(u)=x$, and $U \subset \mathbb{R}^{n}$ an open set. Regard $g$ as a mapping from $U$ to $\mathbb{R}^{m}$ so that the derivative

$$
d g_{u}: \mathbb{R}^{n} \mapsto \mathbb{R}^{m}
$$

is defined. Define $T M_{x}$ to be the image $d g_{u}\left(\mathbb{R}^{n}\right)$ of $d g_{u}$. Intuitively, we think of the tangent space as a hyperplane tangent to $M$ at $x$, for example the plane tangent to the sphere $S^{2}$ at $x$.

The derivative of a mapping is its best linear approximation, so the derivatives are used to specify the linear space $T M_{x}$ which best approximates a manifold $M$ at a point $x$. It is easy to show (Milnor $[1965$, p. 5]) that the definition is independent of the parameterization. Hence, $T M_{x}$ is well defined.

\subsection{Immersions and hypersurfaces}

For the inverse function theorem to apply to $f: M^{n} \mapsto N^{k}$ the dimensions $n$ and $k$ must be equal. If $n<k$, then we can demand that $d f_{x}: T M_{x} \mapsto$ $T M_{f(x)}$ be injective, in which case we say that $f$ is an immersion at $x$. If $f$ is an immersion at every point $x$ then $f$ is an immersion. In addition, if $f$ is a homeomorphism onto $f(M) \subseteq N$, where $f(M)$ has the subspace 
topology induced from $N$, then we say that $f$ is an embedding. If $M \subseteq N$ and the inclusion $i: M \mapsto N$ is an embedding, we say $M$ is a submanifold of $N$. If $f: M^{n} \mapsto N^{k}$ is an immersion, then $n \leq k$ and the difference $k-n$ is the codimension of the immersion $f$. (For examples, see Do Carmo [1992, p. 11].) A hypersurface of a manifold is a submanifold of codimension one, namely smoothly embeds in Euclidean space of one dimension higher. Nabutovsky and Weinberger tend to construct hypersurfaces because certain results, like Smale's Theorem 4.5, work for homeomorphisms but not for diffeomorphisms unless the manifold is a hypersurface.

\subsection{Fundamental groups and homology groups}

Poincaré [1895] was the first to construct an algebraic group which is a topological invariant of the space $Y$ to which it is associated, called the fundamental group, or first homotopy group. Let $Y$ be a topological space, and let $y_{0}$ be a point in $Y$. The $y_{0}$-neighborhood of curves in $Y, C\left(Y, y_{0}\right)$ is the collection of all continuous mappings $f: I^{1} \mapsto Y$ of the unit interval into $Y$ such that $f(0)=f(1)=y_{0}$. Given $f$ and $g$ in $C\left(Y, y_{0}\right), f$ is homotopic to $g$ modulo $y_{0}$ if $f$ can be continuously transformed into $g$, namely there exists a continuous map $h: I^{1} \times I^{1} \mapsto Y$ such that $h(x, 0)=f(x), h(x, 1)=g(x)$ for all $x \in I^{1}$, and $h(0, t)=y_{0}=h(1, t)$ for all $t \in I^{1}$. The equivalence classes form the fundamental group of $Y$ modulo $y_{0}$. It can be shown that this is independent of the choice of $y_{0}$, so it is simply called the fundamental group of $Y$, and written $\pi_{1}(Y)$. If the fundamental group of $Y$ is the identity, then $Y$ is called simply connected.

The fundamental group of the circle $S^{1}$ in Euclidean 2 -space is $(\mathbb{Z},+)$, the group of the integers under addition, and the fundamental group of the torus $T^{2}$ in 3 -space is $(\mathbb{Z} \oplus \mathbb{Z},+)$ because it has two generators. The fundamental group of the standard sphere $S^{n}$ for $n \geq 2$ is the identity because any curve on $S^{n}$ can be shrunk to a point. This property of the fundamental group of $S^{n}$ is the key feature used by Novikov and by Nabutovsky and Weinberger.

Homology groups are easier to compute than homotopy groups but more difficult to define. We refer the reader to a reference on topology but give this intuitive example. Picture the torus $T^{2}$ in Euclidean 3-space lying flat like a donut. Make two vertical slices. These produce two circles $f$ and $g$ in cross section. We say that $f$ and $g$ are homologous because they bound a region, namely that portion of the surface of the torus between then. Now make a horizontal cut through the center of the torus to produce another circle $h$, and note that $f$ and $h$ are not homologous because they do not bound a two dimensional portion of the torus. Homology groups are computed by taking 
equivalence classes under this equivalence relation.

\subsection{The Generalized Poincaré Conjecture}

Definition 4.3 (i) A homology n-sphere is an $n$-manifold $M^{n}$ with homology groups all isomorphic to those of the $n$-sphere $S^{n}$.

(ii) A homotopy $n$-sphere is a homology $n$-sphere $M^{n}$ whose fundamental group $\pi_{1}\left(M^{n}\right)$ is isomorphic to that of $S^{n}$.

Conjecture 4.4 (The Poincaré Conjecture) A compact simply connected 3-manifold without boundary is a 3-sphere. (Namely, a homotopy 3-sphere is homeomorphic to the 3-sphere.)

This famous conjecture is still open for dimension 3, but has been solved for dimension $n \geq 4$.

Theorem 4.5 (Generalized Poincaré Conjecture) (i) (Smale [1961], Stallings [1960]) For all $n \geq 5$ a homotopy $n$-sphere $M^{n}$ is homeomorphic to the n-sphere.

(ii) (Freedman [1982]) Part (i) holds also for $n=4$.

Nabutovsky and Weinberger [NW1, p. 26] use the following theorem.

Theorem 4.6 Let $S$ be smooth n-homotopy sphere $S$ which is also a hypersurface (i.e., smoothly embeds in $\mathbb{R}^{n+1}$ ). Then $S$ is homeomorphic to $S^{n}$ iff $S$ is diffeomorphic to $S^{n}$.

Proof sketch (Weinberger). Milnor [1965b] proves as a corollary to hcobordism that if $\mathrm{X}$ is a contractible manifold with simply connected boundary (and high enough dimension) then $\mathrm{X}$ is diffeomorphic to a disk, and a fortiori, the boundary is diffeomorphic to the sphere.

If a homotopy sphere is embedded in Euclidean space, then it separates it into 2 components: the closure of one of these is compact, which one can check (using Alexander duality, the Whitehead theorem, and Van Kampen's theorem - see Spanier's basic text on Algebraic topology) is contractible. Since the boundary, namely the homotopy sphere, is simply connected, this closure is a disk, and the homotopy sphere is diffeomorphic to the sphere. Note that the Whitehead theorem plus the Hurewicz isomorphism theorem (see Spanier) tell us that a homology $n$-sphere, $n>1$, is a homotopy sphere iff it is simply connected. 
Smale's Theorem 4.5 (i) does not necessarily hold with "diffeomorphic" in place of "homeomorphic," because for all $n \geq 7$ there are differential manifolds homeomorphic to $S^{n}$ but not diffeomorphic to $S^{n}$. These are called exotic spheres. Therefore, some further hypothesis such as being a hypersurface is required in addition to the hypothesis in Theorem 4.5 (i) to achieve a diffeomorphism, which is why Theorem 4.6 is necessary. See Kervaire and Milnor [1963] for work on groups of homotopy spheres, and then Levine [1985] for further work there.

\subsection{Connected sum}

To take the connected sum of two manifolds $X^{2}$ and $Y^{2}$ just cut a small piece from each and join them at the pieces removed. For higher dimensions do the same but pay attention to the orientation.

Definition 4.7 Let $X \# Y$ denote the connected sum of $X$ and $Y$.

Theorem 4.8 Fix $n \geq 5, M$ a smooth manifold of dimension $n$ and $P a$ hypersurface of dimension $n$. Then

$$
(M \# P) \approx_{\text {diff }} M \quad \Longleftrightarrow \quad P \approx_{\text {diff }} S^{n} .
$$

One direction is easy. If $P$ is not simply connected (i.e., has nontrivial fundamental group) then $(M \# P) \not \succsim$ diff $M$.

\section{Riemannian geometry}

For Riemannian geometry, see Do Carmo [1992] and Petersen [1962]. Riemannian geometry developed out of the differential geometry of surfaces $S \subset \mathbb{R}^{3}$. Given a surface $S \subset \mathbb{R}^{3}$, we can measure the lengths of vectors tangent to $S$ at a point $x$ by taking the inner product $\langle u, v\rangle$. To compute the length of a curve, by definition, we integrate its velocity vector (as below). The inner product also allows us to measure the area of domains in $S$, and the angle between curves, as well as to define special curves called geodesics discussed later, along which the length of the curve between points $x$ and $y$ sufficiently close together is less than or equal to the length of any other curve joining $x$ and $y$. Note that the inner product at each point $x \in S$ yields a symmetric bilinear form $I_{x}$, defined in the tangent plane, $T S_{x}$, by $I_{x}(v)=\langle v, v\rangle, v \in T S_{x}$. 


\subsection{Riemannian metrics}

We now wish to extend these definitions to more general smooth manifolds which will be assumed to be Hausdorff spaces with countable bases. (See Do Carmo [1992, pp. 35-45], and Peterson [1998, pp. 2-3].)

Definition 5.1 A Riemannian manifold (or Riemannian structure) $(M, g)$ consists of a smooth manifold $M$ and an inner product (i.e., a symmetric, bilinear, positive-definite form) $g_{p}$, sometimes written $\langle., .\rangle_{p}$, on the tangent spaces $T M_{p}$ for every point $p \in M$. We also assume that $g_{p}$ varies smoothly. That is, for any two smooth vector fields $X$, and $Y$, the inner product $g_{p}(X, Y)$ must be a smooth function of $p$. This is equivalent to the following condition. If $f: U \subset \mathbb{R}^{n} \mapsto M$ is a system of coordinates around $p \in M$, with $f\left(x_{1}, x_{2}, \ldots x_{n}\right)=q \in f(U)$, and if $\frac{\partial}{\partial x_{i}}(q)=d f_{q}(0, \ldots, 1, \ldots, 0)$, then $\left\langle\frac{\partial}{\partial x_{i}}(q), \frac{\partial}{\partial x_{j}}(q)\right\rangle_{q}=g_{i, j}\left(x_{1}, \ldots, x_{n}\right)$ is a differentiable function on $U$. In this case, we call $g$ a Riemannian metric on $M$. Given $\left(x_{1}, x_{2}, \ldots, x_{n}\right)$ we think of $\left(g_{i, j}\left(x_{1}, \ldots, x_{n}\right)\right)$ as a matrix corresponding to the linear map on $T M_{y}$. We call $g_{i, j}$ the local representation of the Riemannian metric (or the $g_{i, j}$ of the metric) in the coordinate system $f: U \subset \mathbb{R}^{n} \mapsto M$.

The most important Riemannian manifold is Euclidean space $\left(\mathbb{R}, g_{\text {euclid }}\right)$ with $\frac{\partial}{\partial x_{i}}$ identified with $e_{i}=(0, \ldots, 1, \ldots 0)$. The metric $g_{\text {euclid }}$ is given by $\left\langle e_{i}, e_{j}\right\rangle=\partial_{i, j}$. It is clear that the metric is independent of $p$.

\subsection{Isometries}

Let $(M, g)$ and $(N, h)$ be Riemannian manifolds and fix a diffeomorphism $f: M \mapsto N$.

Definition 5.2 (i) $f$ is an isometry if:

$$
(\forall p \in M)\left(\forall u, v \in T M_{p}\right)\left[\langle u, v\rangle_{p}=\left\langle d f_{p}(u), d f_{p}(v)\right\rangle_{f(p)}\right] .
$$

(ii) If $f:(M, g) \mapsto(N, h)$ is a diffeomorphism of Riemannian manifolds, define the induced mapping (or pull back) $f^{*} h$ on Riemannian metrics by:

$$
f^{*} h(u, v)_{p}=h(d f(u), d f(v))_{f(p)} .
$$

Note that $f$ is an isometry iff $f^{*} h=g$.

Recall that the set of diffeomorphisms of a manifold $M$ forms a group, called $\operatorname{Diff}(M)$. The main interest is not in the space of metrics on $M$ 
but rather in its quotient by $\operatorname{Diff}(M)$. Isometries play a key role in the Nabutovsky Weinberger results since in $\operatorname{Met}(M)=\operatorname{Riem}(M) / \operatorname{Diff}(M)$ (see $\S 7)$ Nabutovsky and Weinberger take the space of Riemannian metrics on $M$ modulo isometries, i.e., modulo the group of diffeomorphisms $\operatorname{Diff}(M)$. The Gromov-Hausdorff metric will not work on $\operatorname{Met}(M)$ unless we identify Riemannian metrics under isometries.

\subsection{Immersed manifolds}

Let $f: M^{n} \mapsto N^{n+k}$ be an immersion (as defined in $\S 4.4$ ). If $N$ has a Riemannian structure (say with Riemannian metric $h$ ), then $f$ induces a Riemannian metric $g=f^{*} h$ on $M$ by the pullback $f^{*}$ as in Definition 5.2. Namely, $g(u, v)_{p}=\langle u, v\rangle_{p}$ defined by

$$
\langle u, v\rangle_{p}=h\left(d f_{p}(u), d f_{p}(v)\right)_{f(p)} .
$$

Since $d f_{p}$ is injective, $g$ is positive definite. This metric $g$ is called the metric induced by $f$, and we say that $f$ is an isometric immersion.

\subsection{The length of paths in $M$}

Definition 5.3 Given a Riemannian manifold $(M, g)$ we use the Riemannian metric $g(u, v)=\langle u, v\rangle$ to define the length of a $C^{2}$-path $\gamma:[a, b] \mapsto M$ as follows. Let $\dot{\gamma}(t)=\frac{d \gamma}{d t}$, the velocity vector. Define

$$
\ell(\gamma)=\int_{a}^{b}\langle\dot{\gamma}(t), \dot{\gamma}(t)\rangle_{\gamma(t)}^{\frac{1}{2}} d t
$$

in which case we call $\langle\dot{\gamma}(t), \dot{\gamma}(t)\rangle^{1 / 2}$ the velocity or speed of $\gamma$ at $t$.

Definition 5.4 Define a metric space metric $d: M \times M \mapsto \mathbb{R}$ as follows:

$$
d(x, y)=\inf \left\{\ell(\gamma): \gamma \text { a } C^{2} \text { path from } x \text { to } y\right\} .
$$

Note that this metric satisfies the usual metric space metric properties:

1. $d(x, x)=0$,

2. $d(x, y)=d(y, x)$,

3. $d(x, y)+d(y, z) \leq d(x, z)$ [triangle inequality]

This is the metric space metric on $M$ which is induced by the Riemannian metric $g$. 
Definition 5.5 Given a compact Riemannian manifold $(M, g)$ let $d(x, y)$ be the metric space metric as defined in Definition 5.4. Define the diameter of $(M, g)$ to be

$$
D((M, g))=\max \{d(x, y): x, y \in(M, g)\} .
$$

In the space $\operatorname{Met}(M)$ considered by Nabutovsky and Weinberger in $\S 7$, the points will be metrics on $M$ modulo diffeomorphisms. Each such metric $(M, g)$ on the compact manifold $M$ will have associated with it a diameter $D((M, g))$ as just defined in Definition 5.5. The local minima in the space Met $(M)$ discussed in $\S 7$ and $\S 9$ will all be taken with respect to the value of the diameter function on these manifolds.

\section{Unsolvable fundamental groups and geodesics}

\subsection{The covariant derivative and geodesics}

Let $S \subset \mathbb{R}^{3}$ be a surface and let $c: I \mapsto S$ be a parametrized curve in $S$ with $V: I \mapsto \mathbb{R}^{3}$ a vector field along $c$ tangent to $S$. The vector $\frac{d V}{d t}(t)$, $t \in I$, does not always belong to the tangent plane of $S, T S_{c(t)}$. To correct this, geometers define the orthogonal projection of $\frac{d V}{d t}(t)$ on $T S_{c(t)}$. This is the covariant derivative and is denoted by $\frac{D V}{d t}(t)$. (See Do Carmo [1992, pp. 48-50] and Petersen [1998].)

Definition 6.1 A parametrized curve $\gamma: I \mapsto M$ is a geodesic at $t_{0} \in I$ if $\frac{D}{d t}\left(\frac{d \gamma}{d t}\right)=0$ at the point $t_{0}$, where $\frac{D}{d t}$ is the covariant derivative described above. If $\gamma$ is a geodesic at $t$ for all $t \in I$ we say $\gamma$ is a geodesic.

Intuitively, a geodesic is a curve with zero acceleration, hence with constant velocity. The concept of geodesic is a curve which minimizes the distance between two nearby points. For surfaces in $\mathbb{R}^{3}$ the geodesics are those curves $\gamma(s)$ for which the acceleration $\gamma^{\prime \prime}(s)$ in $\mathbb{R}^{3}$ is perpendicular to the surface (and hence from the viewpoint of the surface is zero). For example, the geodesics on the sphere $S^{2}$ are great circles since these minimize distance between two nearby points.

\subsection{The Fundamental Group and Geodesics}

The following theorem by Gromov gives a powerful connection between computability properties (the unsolvability of the fundamental group) and geometric properties (the number of closed, contractible geodesics). 
Theorem 6.2 (Gromov (1981)) Let $M$ be a smooth manifold whose fundamental group has an unsolvable word problem. Then there exist infinitely many contractible, closed geodesics on $M$.

Nabutovsky [1996c] gives a proof, and Nabutovsky and Weinberger $[\mathrm{NW} 2, \S 5]$ give a proof sketch under the title "A toy problem," because it foreshadows their work. The idea is that if $M$ is a manifold with fundamental group $\pi$ and no contractible geodesics, then we can solve the word problem for $\pi$ as follows. For any word in the generators of $\pi$, construct a simple closed curve representing that word in the fundamental group, and apply curve shortening. If in the asymptotic process one obtains a closed curve of positive length, then it must be nontrivial, but if it continually shortens to small length, then it is contractible. If there are only finitely many closed, contractible geodesics, then this gives the solvability of $\pi$.

\subsection{Curvature}

For a discussion of curvature see Guillemin and Pollack, pages 75 and 195, and Do Carmo pages 94, 131, and 162. For example, the curvature of the round 2 -sphere of radius $r$ in Euclidean 3 -space is $1 / r^{2}$ everywhere. As the radius increases, the curvature decreases, and large spheres are flatter than small ones.

In higher dimensions one usually considers sectional curvature, a more complex notion presented in Chavel [1993, pp. 49-100]. Nabutovsky and Weinberger will assume (in Definition 7.1) that the manifold $M$ has sectional curvature $|K| \leq 1$ for the reasons explained in $\S 9$. They wish to study the local minima of the diameter function on $A \ell(M)$ as defined in Definition 7.3. Any Riemannian metric $(M, g)$ may be rescaled to $(M, h)$ by simply defining $h(x, y)=\lambda g(x, y)$ for $\lambda \in \mathbb{R}$. Hence, by continually rescaling we might not get a local minimum at all. However, in this case the sectional curvature $K_{h}=\left(1 / \lambda^{2}\right) K_{g}$. Hence, the curvature hypothesis $|K| \leq 1$ limits the amount of rescaling and allows local minima to exist. The hypothesis $|K| \leq 1$ is a weak assumption and roughly says (up to rescaling) that $K$ is uniformly bounded away from $I_{\infty}$.

\section{The Geometric Space Riem/Diff}

\section{$7.1 \quad \operatorname{Met}(M)$}

Definition 7.1 Fix a smooth, compact, manifold $M$ of dimension $n \geq 5$ and sectional curvature $|K| \leq 1$. (See the explanation in §6.3.) Define 
$\operatorname{Riem}(M)$ to be the space of Riemannian metrics on $M$ (as in $\S 5.1$ ), and define

$$
\operatorname{Met}(M)=\operatorname{Riem}(M) / \operatorname{Diff}(M)
$$

the space of Riemannian metrics on $M$ modulo diffeomorphisms which are isometries (as in §5.2). Met stands for "metrizable."

It is key that we take $\operatorname{Riem}(M)$ modulo isometries because the GromovHausdorff metric $d_{G H}$ defined in $\S 7.2$ will not work without identifying isometric elements. Note that we may regard $\operatorname{Riem}(M)$ as a subspace of the space $\mathcal{M}$ of all compact metric spaces by identifying $(M, g)$ of Riem with $\left(M, d_{M}\right)$ where $d_{M}$ is the length metric on $M$ coming from $g$. However, $\mathcal{M}$ is not complete with respect to the Gromov-Hausdorff metric defined below.

To foreshadow later ideas, note that if a smooth hypersurface in Euclidean space is diffeomorphic to, say, the sphere $S^{n}$, then it defines a welldefined element of $\operatorname{Met}\left(S^{n}\right)$, with the metric induced by Euclidean space. However, one would need the specific diffeomorphism between that hypersurface and the sphere to "lift" that element to Riem $\left(S^{n}\right)$. Later on Nabutovsky and Weinberger produce interesting sets of hypersurfaces, and hence of Riemannian metrics. The key is, of course, that they will not be producing them as $g$ 's, i.e., by explicitly writing down a Riemannian metric $g$, but rather as hypersurfaces in Euclidean space.

\subsection{The Gromov-Hausdorff metric}

Hausdorff had defined the classical distance between two subsets of a metric space, and in 1980 Gromov improved upon it to get the following definition. We call this the Gromov-Hausdorff distance between two abstract metric spaces. See Peterson [1993, p. 490].

Definition 7.2 [Gromov-Hausdorff metric] (i) For compact $A, B \subseteq$ metric space $C$, define

$$
d_{G H}(A, B ; C)
$$

to be the least $x$ such that every point of $A$ is within distance $x$ of some point of $B$ and vice versa.

(ii) For $A, B$ abstract metric spaces their distance

$$
d_{G H}(A, B)
$$

is the infimum over all metric spaces $C$ containing $A$ and $B$ isometrically. 
Note that if $d_{G H}(X, Y)=0$ then $X$ and $Y$ are isometric. Hence, we must consider $\operatorname{Riem}(M)$ modulo isometries for $d_{G H}$ to work, because we must identify isometric spaces. Also any two Riemannian manifolds of diameter $\leq x$ are $2 x$-close to each other in the $d_{G H}$ metric.

\subsection{A path metric on $A \ell(M)$}

The authors Nabutovsky and Weinberger work not with $\operatorname{Met}(M)$ itself but rather with the following well behaved subset $A \ell(M)$.

Definition 7.3 Define $A \ell(M)$ to be the subset of $\operatorname{Met}(M)$ consisting of the closure in the Gromov-Hausdorff metric of isometry classes of metrics (in $\operatorname{Riem}(M) / \operatorname{Diff}(M)$.

Nabutovsky and Weinberger do not work with the Gromov-Hausdorff metric directly but prefer to use the path metric on $A \ell(M)$ defined as follows in Nabutovsky-Weinberger [NW]. Furthermore, they work not with zero, but with a very small constant $\epsilon>0$ which depends on Cheeger's work [1970], and all their estimates of local minima, and so on, are as functions of $\epsilon$.

Definition 7.4 The distance between two metrics $\mu_{1}$ and $\mu_{2}$ on $M$ is defined as the length of the shortest path connecting these metrics in $A \ell(M)$. Namely, define this as

$$
d\left(\mu_{1}, \mu_{2}\right)=\inf \Sigma_{1 \leq i<k} d_{G H}\left(\nu_{i}, \nu_{i+1}\right),
$$

where $\mu_{1}=\nu_{1}, \ldots, \nu_{k}=\mu_{2}$ is a sequence of metrics in $A \ell(M)$ such that for any $i<k, d_{G H}\left(\nu_{i}, \nu_{i+1}\right) \leq \epsilon$, and the infimum is taken over all such finite sequences and all $\epsilon>0$. From now on we use $\operatorname{Met}(M)$ to mean its completion $A \ell(M)$ and we use $d(\mu, \nu)$ to mean the path metric defined here.

Note that this same conversion from a metric to a path metric on the same space works for any metric space metric $d$ not only the GromovHausdorff metric $d_{G H}$. Nabutovsky and Weinberger introduce the path metric, rather than simply using $d_{G H}$ under which the space $\operatorname{Riem}(M) / \operatorname{Diff}(M)$ is already complete. The reason is that if we have a manifold, like the sphere $S^{2}$, we may have a metric $\hat{d}$ which measures distances between antipodal points $x$ and $y$ along a diameter. In this case, we might have $\hat{d}(x, y)=d_{1}$, the length of the diameter, but have no path in $S^{2}$ from $x$ to $y$ which has length $d_{1}$. Using the path metric $d(x, y)$ guarantees that if $d(x, y)=d_{1}$ then there is a path of length $d_{1}$ on the manifold $M$ from $x$ to $y$. 


\subsection{Local minima on $\operatorname{Met}(M)$}

Definition 7.5 Let $f: X \mapsto[0, \infty)$ and $x, y \in X$.

(i) $\pi(x, y)$ denotes an arbitrary path from $x$ to $y$ such that $f(y)<f(x)$.

(ii) Define the rise of the path,

$$
\rho(\pi(x, y))=\max \{f(z)-f(x): z \text { on } \pi(x, y)\} .
$$

Namely, $\rho$ represents how much higher above $x$ you must climb to traverse the path to $y$.

(iii) If $x$ is a local minimum for $f$, define the depth of $x$,

$$
\delta(x)=\min \{\rho(\pi(x, y)): \text { all } \pi(x, y): f(y)<f(x) \& y \neq x\} .
$$

Hence, $\delta(x)$ is the minimum altitude over $d_{x}$ to reach any local minimum $y$ below $x$ by any path. This has the usual intuitive meaning. Picture a mixing bowl with sides of height four above the bottom, but one side lowered to three inches for easier pouring. The bowl would hold three inches of water, and that is its depth by this definition.

\subsection{Sublevel sets}

Definition 7.6 Let $f: X \mapsto[0, \infty)$ be any function. For any positive real $x$ the set $f^{-1}([0, x])$ is called a sublevel set of $f$.

In the following results on sublevel sets Nabutovsky and Weinberger always take $X=\operatorname{Riem} / \operatorname{Diff}(M)$ and take $f(x)$ to be $D(x)$, the diameter function, defined in Definition 5.5.

If all sublevel sets of $f$ are compact and $f$ is continuous, then $f$ has a local minimum on every connected component of every sublevel set. These local minima on connected components of sublevel sets are local minima of $f$ on $X$. Indeed, the value of $f$ at any point outside a sublevel set is greater than the value of $f$ at any point of the sublevel set.

The component of the sublevel set $f^{-1}((0, D(x)+\delta(x)))$ which contains $x$ is regarded as a basin containing $x$. It has the absolute minimum of $D(x)$ restricted to this component as the basin's minimum, and the basin has depth at least $\delta(x)$. Typically $\delta(x)$ is much larger than $D(x)$ and we can intuitively think of $x$ as lying "near the bottom" of the basin. If $x$ is a global minimum, then the whole space is the corresponding basin of infinite depth. See $[N W 2$, p. 1]. 


\section{Computability results for differential geometry}

In this section we give the definition and statement of the computability results to be applied to differential geometry. The proofs of the computability results in this section will appear in Csima and Soare [ta]. Theorem 8.12 was announced by Soare in answer to the question posed by Nabutovsky and Weinberger. Later in her Ph.D. thesis under Soare, Csima [2003] strengthened this to Theorem 8.14, and developed a number of other properties of the dominating ordering relation $A \succ B$ defined in Definition 8.11.

\subsection{Standard definitions}

The following standard notions of computability theory can be found in [Soare, 1987].

Definition 8.1 (i) Let $\left\{P_{e}\right\}_{e \in \omega}$ be an effective indexing of all Turing programs.

(ii) Define $\varphi_{e}(x)=y$ if $P_{e}$ with input $x$ eventually halts with output $y$.

(iii) Define $\varphi_{e, s}(x)=y$ if $\varphi_{e}(x)=y$ in $<s$ steps of $P_{e}$ and $x, y, e<s$.

(iv) Let $W_{e}=\operatorname{domain}\left(\varphi_{e}\right)$, the $e^{t h}$ computably enumerable (c.e.) set. We refer to $\left\{W_{e}: e \in \omega\right\}$ as the standard listing or Kleene listing of all computably enumerable (c.e.) sets.

(v) $W_{e, s}=\operatorname{domain}\left(\varphi_{e, s}\right)$, the approximation to $W_{e}$ at stage $s$.

(vi) Two sets $A$ and $B$ (or functions) have the same (Turing) degree if $A$ is computable from $B\left(A \leq_{\mathrm{T}} B\right)$ and $B \leq_{\mathrm{T}} A$.

(vii) The Halting Problem is the c.e. set $K_{0}=\left\{\langle e, x\rangle: x \in W_{e}\right\}$. (Hence, $W_{e} \leq_{\mathrm{T}} K_{0}$ for every $e$, so $K_{0}$ has the greatest degree, denoted by boldface $\mathbf{0}^{\prime}$, among c.e. sets.

(viii) $A\lceil n=A \cap[0, n)$, the restriction of the set $A$ to integers $\{k: k<n\}$, and similarly define the restriction $f\lceil n$ of a function to $[0, n)$.

Essential to the NW results is the following settling function.

Definition 8.2 (i) For each $W_{e}$ define the partial computable halting function,

$$
\theta_{e}(x)= \begin{cases}(\mu s)\left[x \in W_{e, s}\right] & \text { if } s \text { exists } \\ \uparrow & \text { otherwise. }\end{cases}
$$


(ii) For every $W_{e}$ define the settling function:

$$
\begin{aligned}
\sigma_{e}(x) & =(\mu s)\left[W _ { e , s } \left\lceilx=W_{e}\lceil x],\right.\right. \text { namely } \\
\sigma_{e}(x) & =\max \left\{\theta_{e}(y): y<x \quad \& \quad \theta_{e}(y) \downarrow\right\} .
\end{aligned}
$$

(This is also called the computation function in Soare [1987, p. 99].)

Proposition 8.3 For all $e \in \omega, W_{e} \equiv_{\mathrm{T}} \sigma_{e}$.

Note that, although $\sigma_{e}$ clearly has the same Turing degree as the c.e. set $W_{e}$, its graph will generally not be a c.e. set, i.e., it will not be a computable function. Furthermore, if $W_{e}=K_{0}$, then $\sigma_{e}$ will have degree $\mathbf{0}^{\prime}$.

Remark 8.4 Note that the settling function is not defined for a c.e. degree or even for a c.e. set such as $K_{0}$. It is only defined when we have a specific index for a specific c.e. set $A$. Other indices for $A$ can have vastly different settling functions even though these are all Turing equivalent. We regard $e$ for $W_{e}=A$ as a specific algorithm (among many) to enumerate $A$, and the settling time $\sigma_{e}(x)$ measures speed of convergence of this algorithm. We never write $\sigma_{A}(x)$ unless we have in mind a particular index $e$ such that $W_{e}=A$. Likewise, $\sigma_{\mathbf{d}}$ is not defined for a c.e. degree $\mathbf{d}$, but only for some specific $W_{e} \in \mathbf{d}$. However, there is a sense in which local minima in geometry can have depths and density related to a c.e. degree as we explain in $\S 9$.

\subsection{Dominating the settling time}

Nabutovsky and Weinberger [NW2] used the following partial orderings on c.e. sets defined in terms of domination properties.

Definition 8.5 (i) $\quad A \succ B$ iff $\left(\exists W_{i}=A\right)\left(\exists W_{j}=B\right)$

$$
(\forall \text { computable } f)(\text { a.e. } x)\left[\sigma_{i}(x)>f\left(\sigma_{j}(x)\right)\right] .
$$

(ii) $A \succ \succ B$ iff $\left(\forall W_{i}=A\right)\left(\forall W_{j}=B\right)$ we have equation (3).

Definition 8.6 $A \leq_{\mathrm{ibT}} B$ if $A<_{\mathrm{T}} B$ by a computation with use $u(x) \leq x$.

(The notation $A \leq_{b T} B$ means that $A$ is bounded Turing reducible to $B$, namely Turing reducible by a procedure in which the computation on input $x$ is bounded by a computable function $f(x)$. The notation $i b T$ suggests that the bounding function is the identity $f(x)=x$. Here $u(x)$ denotes the largest integer used by the oracle Turing machine to compute $A$ from $B$ on $\operatorname{argument} x$.) 
Theorem 8.7 (Csima)

$$
\begin{array}{lccc}
{\left[W_{e} \leq_{i b T} W_{i} \quad \& \quad W_{i} \prec W_{k}\right]} & \Longrightarrow \quad W_{e} \prec W_{k} \\
{\left[W_{e} \preceq W_{i} \quad \& \quad\right.} & \left.W_{i} \leq_{i b T} W_{k}\right] & \Longrightarrow & W_{e} \prec W_{k} .
\end{array}
$$

Corollary 8.8 (Nies) $B \prec A$ iff $B \prec \prec A$.

Csima's theorem implies that the ordering $\prec$ is well defined on c.e. sets.

\section{Corollary 8.9 (Csima and Nies, Transitivity)}

$$
[A=B \quad \& \quad B \prec C] \Longrightarrow \quad A \prec C .
$$

However, Csima [2003] has also proved that it is not well defined on Turing degrees of c.e. sets.

Theorem 8.10 (Csima) Given c.e. sets $A \succ B$, there exists a c.e. set $C \equiv_{T} A$ such that $C \nsucc B$.

\subsection{A dominating sequence}

Definition 8.11 A sequence $\left\{A_{n}\right\}_{n \in \omega}$ of c.e. sets is a dominating sequence if there is a computable function $h(x)$ such that $A_{n}=W_{h(n)}, n \in \omega$, and

$$
(\forall n)\left[W_{h(n)} \succ W_{h(n+1)}\right] .
$$

Namely, for $A$ we take not only the set $W_{h(n)}=A_{n}$ but also its specific index $h(n)$ and we require that

(6) $\quad(\forall$ computable $f)(\forall n)($ a.e. $x)\left[\sigma_{h(n)}(x)>f\left(\sigma_{h(n+1)}(x)\right)\right]$.

Theorem 8.12 (Soare) There is a dominating sequence $\left\{A_{n}\right\}_{n \in \omega}$.

Weinberger originally asked Soare to prove the theorem in this form, Soare did. He then asked Soare to prove it with $\succ \succ$ in place of $\succ$, which Soare also did by a small variation of the original argument. By Theorem 8.7 and Corollary 8.8 this extension is unnecessary since it follows by the original Soare Theorem 8.12. Let $\mathbb{Z}$ denote the set of positive and negative integers. Then Weinberger asked for both an ascending and descending dominating sequence. By the same proof Soare proved Theorem 8.12 in the form with $\mathbb{Z}$ in place of $\omega$. 
Definition 8.13 Fix a monotonically increasing computable function $g$. A sequence $\left\{W_{h(n)}\right\}_{n \in \omega}$ of c.e. sets is a $g$-dominating sequence if for all $n \in \omega$,

$$
(\forall \text { computable } f)(\text { a.e. } x)\left[\sigma_{h(n)}(x)>f\left(\sigma_{h(n+1)}(g(x))\right)\right] .
$$

This is the same as Definition 8.11 except that the elements $A\lceil x$ must "guard" $B\left\lceil g(x)\right.$ not merely $B\left\lceil x\right.$ as before, where $A=W_{h(n)}$ and $B=$ $W_{h(n+1)}$. By a similar proof, Csima extended Soare's Theorem 8.12 in the following form which Weinberger had later requested of Soare because it simplifies some of the proofs in his paper with Nabutovsky.

Theorem 8.14 (Csima) For any monotonically increasing computable function $g$, there is a g-dominating sequence $\left\{W_{h(n)}\right\}_{n \in \omega}$.

\section{The Nabutovsky Weinberger (NW) Results}

The Nabutovsky Weinberger results are very difficult to state precisely. Even in their paper [NW2] they state several versions of one of the main theorems, Theorem 0.1, an intuitive version and later a formal version, and even that version avoids some of the more subtle points. Here we give an intuitive version which relates the geometry to the computability. The best references are [NW1] and [NW2] for the results and Weinberger's Porter lectures [ta] for a global introduction to the subject. We supply a number of definitions, explanations, and details not explicitly given in these papers.

\subsection{C.E. degrees and local minima}

Notation 9.1 Following Nabutovsky and Weinberger [NW2] we let lower case Greek letters, $\alpha, \beta$, and $\gamma$ denote computably enumerable (c.e.) degrees.

Definition 9.2 For notational convenience from now on we call a manifold $M$ suitable if it is smooth, compact, of dimension $n \geq 5$, and of sectional curvature $|K| \leq 1$.

Definition 9.3 (i) If $f$ is a real valued function on a metric space $X$ with metric $m(x, y)$ and $Y \subseteq X$, then $Y$ is $f$-dense if

$$
(\forall x)(\exists y \in Y)[m(x, y) \leq f(x)] .
$$

(ii) If $\alpha$ is a c.e. degree, then $Y$ is $\alpha$-dense if $Y$ is $f$-dense for a function of degree $\alpha$. 
(iii) For $g$ a function from $\omega$ to $\omega$, a set $\left\{N_{k}\right\}_{k \in \omega}$ of local minima on $\operatorname{Met}(M)$ is $g$-deep if $d\left(N_{k}\right) \geq g\left(\left[D\left(N_{k}\right)\right]\right)$ where $d\left(N_{k}\right)$ is the depth of $N_{k}, D$ is the diameter function, and $[r]$ denotes the greatest integer less than the real $r$.

(iv) For $\alpha$ a c.e. degree a set $\left\{N_{k}\right\}_{k \in \omega}$ of local minima on $\operatorname{Met}(M)$ is $\alpha$-deep if it is $g$-deep for some function $g$ of degree $\alpha$.

\section{Theorem 9.4 (NW1, Theorem 0.1, Informal)}

For every suitable $n$-manifold there are infinitely many local minima of the diameter functional on the subset $A \ell(M)$ of $\operatorname{Met}(M)$. Moreover, there is a constant $c(n)$ depending only on $n$ such that for every c.e. degree $\alpha$ the local minima of depth at least $\alpha$ are $\alpha$-dense in the path metric on $A \ell(M)$, and the number of $\alpha$-deep local minima where the diameter does not exceed $d$ is not less than $\exp \left(c(n) d^{n}\right)$.

The authors write that more precisely, this theorem asserts that for any c.e. degree $\alpha$, any c.e. set $A \in \alpha$, and any index $e$ such that $W_{e}=A$ there exist increasing functions $f$ and $g$ equicomputable with the settling function for $W_{e}$ such that for all sufficiently large $d$ there exist at least $\exp \left(c(n) d^{n}\right)$ local minima of the diameter functional on $A \ell(M)$, where the value of the diameter does not exceed $d$, and such that for each of these minima the depth of the graph of diameter corresponding to this minimum is at least $f(d)$. Moreover, such local minima are $g(d)$ dense in a path metric on $A \ell(M)$, and the number of $\beta$-deep local minima where the diameter does not exceed $d$ is not less than $\exp \left(c(n) d^{n}\right)$.

Each possible value $d$ for the diameter functional $D(x)$ determines a sublevel set $D^{-1}([0, d])$ as defined in Definition 7.6, and we look for local mimima where the diameter does not exceed $d$. Informally, each c.e. degree $\alpha$ determines what the authors call a "scale," and there are many distinguished local minima of the diameter functional which are deep at that scale, meaning that the depth of the local minima is equicomputable with a function of degree $\alpha$. Nabutovsky and Weinberger later state this theorem more formally in [NW2, §12].

Theorem 9.5 (NW1, Theorem 0.1, Rigorous version) Let $M$ be a suitable $n$-manifold, let $A$ be any c.e. set and $W_{e}=A$, and let $\sigma_{e}(x)$ be its settling function as defined in Definition 8.2. There is a constant $c(n)$ depending only upon $n$, and increasing unbounded computable functions $f$ and $g,(f<g)$, such that for all sufficiently large $d$ the number of local minima of the diameter functional $D(x)$ on $A \ell(M)$ such that the value of 
diameter does not exceed $d$ and of depth between $f\left(\sigma_{e}(d)\right)$ and $g\left(\sigma_{e}(d)\right)$ is at least $\exp \left(c(n) d^{n}\right)$. Moreover, these $f\left(\sigma_{e}(d)\right)$-deep local minima form a $g\left(\sigma_{e}(d)\right)$-dense in the path metric subset of $D^{-1}((0, x]) \subset A \ell(M)$.

\subsection{The idea of the proof}

The outline of the proof very roughly is this. Fix $n \geq 5$, a suitable $n$-manifold $M$, a c.e. set $A$, an index $e$ such that $W_{e}=A$ and let $\sigma_{e}(x)$ be the settling function for $W_{e}$.

By the Boone-Novikov Theorem 3.1 there is a finitely presented group $H$ whose word problem has the same degree as that of $A$. By the AdjanRabin Triviality Theorem 3.2 we can use $H$ to find a computable sequence of finitely presented groups $\left\{G_{k}\right\}_{k \in \omega}$ such that

$$
k \in A \Longleftrightarrow G_{k} \cong\langle e\rangle .
$$

By Novikov's Theorem 3.7, these groups can be used to construct an effective sequence of $n$-manifolds, $\left\{P_{k}\right\}_{k \in \omega}$ such that

$$
k \in A \Longleftrightarrow P_{k} \approx_{\text {diff }} S^{n} .
$$

Nabutovsky and Weinberger considerably extend these ideas, making use of the deep refinement of the Boone-Novikov theory from Sapir, Birget, and Rips [2002]. They construct a homology sphere in a very careful way using ideas of Clozel from number theory and ideas of Hausmann Vogel from geometric topology to get a homology sphere with computability properties of the word problem. In particular, they build a sequence $\left\{P_{k}\right\}_{k \in \omega}$ of homology spheres, which are also hypersurfaces, and they use the connected sum to obtain the desired objects $N_{k}={ }_{\text {dfn }}\left(M \# P_{k}\right)$. (As described earlier, the $P_{k}$ inherit the metric of Euclidean space.) Now by Smale's Theorem 4.5 and its extension for hypersurfaces Theorem 4.8, we have for every $k$,

$$
N_{k}=\left(M \# P_{k}\right) \approx_{\text {diff }} M \quad \Longleftrightarrow \quad P_{k} \approx_{\text {diff }} S^{n} .
$$

Putting these lines together we have that for every $k$,

$$
k \in A \Longleftrightarrow N_{k} \in \operatorname{Met}(M) .
$$

Hence, Nabutovsky and Weinberger construct from every c.e. set $W_{e}$ an effective sequence of $n$-manifolds $\left\{N_{k}^{e}\right\}_{k \in \omega}$ such that $k \in W_{e}$ iff $N_{k}^{e} \in \operatorname{Met}(M)$. The event " $k \in A$ " is c.e. by definition and the event $N_{k}^{e} \in \operatorname{Met}(M)$ is also c.e. because it depends on the collapse to the identity 
of the group $G_{k}$ which is a c.e. event. The $P_{k}^{e}$ are both homology spheres and hypersurfaces, but the resulting manifolds $N_{k}^{e}=M \# P_{k}^{e}$ are more complicated, and are probably neither.

As $N_{k}^{e}$ is revealed to be a point of $\operatorname{Met}(M)$ a process (called "nondeterministic gradient flow") takes place for finding other points on $\operatorname{Met}(M)$ closely associated with and nearby to $N_{k}^{e}$ which are local minima. Their construction guarantees that these local minima exist in the quantity and depth specified in Theorem 9.5. The authors do not claim that uniformly effectively in $e$ and $k$ they can find the required local minimum $Q_{k}^{e}$, but rather they can produce a set $\mathcal{S}_{k}^{e}$ which contains it. What is uniformly computable in the settling function $\sigma_{e}(k)$ is the estimate of the depth of at least one local minimum $Q_{k}^{e}$ in $\mathcal{S}_{k}^{e}$ as specified in Theorem 9.5. Most of this depth is achieved by the complexity "above" $N_{k}^{e}$ rather than moving "below" $N_{k}^{e}$ to obtain the local minima in $\mathcal{S}_{k}^{e}$.

Nabutovsky and Weinberger also prove that if $k \in W_{e}$, then the distance in $\operatorname{Met}\left(S^{n}\right)$ from $P_{k}^{e}$ to $S^{n}$, the standard sphere, is equicomputable with the settling time $\sigma_{e}(k)$, and this also is equicomputable with the distance function in $\operatorname{Met}(M)$ from $N_{k}^{e}$ to the standard point $M$. The intuitive reason that the depth and distance must be this complicated is very roughly that shallower depths or shorter paths would give a shorter proof of the triviality of a group, and hence a shorter proof of the halting problem for a certain Turing machine.

\subsection{How deep is deep?}

Suppose we wish to compare the depth $d$ of a small basin, such as a bath tub, with the depth $e$ of a giant basin, such as the Grand Canyon. We could say that the exponential $2^{d}$ is still less than $e$. However, when we iterate a few more exponentials this inequality will no longer be true. Nabutovsky and Weinberger wanted a much more impressive comparison of relative depth in that any computable function of the smaller is still less than the larger, at least on almost all arguments. This definition makes sense only if we are dealing with infinite sequences, but their construction above produces just such a infinite sequence $\left\{N_{k}^{e}\right\}_{k \in \omega}$ for each c.e. set $W_{e}$.

Nabutovsky and Weinberger began by using c.e. degrees to make the comparison of relative depths. For example, if $W_{e}<_{\mathrm{T}} W_{i}$ then for any computable function $f$ there are infinitely many arguments $k \in \omega$ at which $f\left(\sigma_{e}(k)\right)<\sigma_{i}(k)$ and hence at which the local minima associated with $N_{k}^{i}$ are vastly deeper than those associated with $N_{k}^{e}$. If $W_{e}<_{\mathrm{T}} W_{i}$ then by the Sacks Density Theorem there exists $W_{j}$ such that $W_{e}<_{\mathrm{T}} W_{j}<_{\mathrm{T}} W_{i}$. 
Hence, for any computable function $f$ there is an infinite set $X$ of arguments $k$ at which $f\left(\sigma_{e}(k)\right)<\sigma_{j}(k)$ and an infinite set of arguments $k$ at which $f\left(\sigma_{j}(k)\right)<\sigma_{i}(k)$, but the sets $X$ and $Y$ need not coincide. To get the depth comparisons they wanted, Nabutovsky and Weinberger asked Soare for a finer measure.

\subsection{Using the settling function}

As in Definition 8.5 Nabutovsky and Weinberger defined for c.e. sets $A$ and $B$ the ordering $A \succ B$ if

$$
\left(\exists W_{i}=A\right)\left(\exists W_{j}=B\right)(\forall \text { computable } f)(\text { a.e. } x)\left[\sigma_{i}(x)>f\left(\sigma_{j}(x)\right)\right] .
$$

By Nies's Corollary 8.8 this does not depend on the choice of $i$ and $j$.

If we apply Theorem 9.5 to c.e. sets $W_{i} \succ W_{j}$ then the sequences $\left\{N_{k}^{i}\right\}_{k \in \omega}$ and $\left\{N_{k}^{j}\right\}_{k \in \omega}$ will have the property that the associated local minima of the former are vastly deeper almost everywhere than those of the latter.

At Weinberger's request Soare constructed in Theorem 8.12 the dominating sequence $\left\{A_{n}\right\}_{n \in \mathbb{Z}}$ such that $A_{n} \succ A_{n+1}$. Applying Theorem 9.5 to the Soare sequence $\left\{A_{e}\right\}_{e \in \mathbb{Z}}$ we get a sequence of manifolds such that the local minima determined by $A_{e}$ are vastly deeper than those determined by $A_{e+1}$. As the ideas in the Nabutovsky-Weinberger theory developed, the correspondences became sharper. For example, in Theorem 9.4 the depth and density of the local minima corresponding to the sequence $\left\{N_{k}^{e}\right\}_{k \in \omega}$ are only correlated with some function in Turing degree of $W_{e}$, while in Theorem 9.5 they are correlated more precisely with the settling function $\sigma_{e}(x)$. Notice that in the Soare sequence $\left\{A_{n}\right\}_{n \in \mathbb{Z}}$ we do not claim that $A_{n}>_{\mathrm{T}} A_{n+1}$ in Turing degree, but merely that $A_{n} \succ A_{n+1}$.

However, it is not only the depth of the local minima which is important but also their distribution and proximity to one another. The property in Theorem 9.5 that the local minima are form a $g\left(\sigma_{e}(d)\right)$-dense in the path metric subset of $D^{-1}((0, x]) \subset A \ell(M)$ implies the various local minima are relatively close together. For example, this means that there are very deep local minima with medium size local minima coming off their sides, with very small local minima coming off their sides and so forth. This gives justification for the word "fractal" in the title of [NW2]. To express this intuition Nabutovsky and Weinberger [NW2, §12] wrote:

PANORAMIC VIEW OF THE GRAPH OF DIAMETER ON $A \ell(M)$. There are 'pits' or 'basins' in the graph of diameter with depth of the magnitude roughly equal to the 'halting function' 
[i.e., settling function] for $A_{2}$ and spaced at intervals growing slightly faster than their depth. These are merely bumps in the basins of the (spaced much further apart) much deeper basins that correspond to $A_{1}$. And even these huge basins are merely bumps in the basins corresponding to $A_{0}$. And so on.

\subsection{The simplicial norm and lower bounds on volume}

The next theorem is formally contained in Theorem 9.5. However, studying the proof sheds light on another aspect of how computability is applied in differential geometry, in this case to show the existence of even a single local minimum on $\operatorname{Met}(M)$ where $M$ is, say, the torus $T^{5}$. Here the statement of the theorem requires no mention of sets or degrees, but no proof is known which avoids computability.

Theorem 9.6 (Nabutovsky-Weinberger) For any smooth compact manifold $M^{n}, n \geq 5$, there are infinitely many extremal metrics $g$ on $M$ in the sense that:

1. $|K(g)| \leq 1$, and

2. Any nearby metric $h$ on $M$ has either $|K(h)|>1$ at some point or $D(h)>D(g)$.

After stating Gromov's Theorem 9.8 below relating lower bounds on Ricci curvature to such bounds on volume in the presence of an estimate on simplicial norm, Nabutovky and Weinberger prove the following theorem.

Theorem 9.7 (NW2, Theorem 9.2) For every $n \geq 5$, there is an effective sequence $\left\{P_{k}\right\}_{k \in \omega}$ of homology spheres such that for every $k$ either:

1. $P_{k}$ is diffeomorphic to $S^{n}$ (written $P_{k} \approx$ diff $S^{n}$ ), or

2. the simplicial norm $\left\|P_{k}\right\|>1$.

Furthermore,

$$
\begin{aligned}
& S=\{k: \text { case }(1) \text { holds }\} \text { is computably enumerable, and } \\
& T=\{k: \text { case }(2) \text { holds }\} \text { is not computably enumerable. }
\end{aligned}
$$

and $S \cap T=\emptyset$. 
We will be using manifolds of the form $M \# P_{k}$ to refine Novikov's theorem to learn that even in the presence of a simplicial norm estimate one cannot decide whether a smooth manifold is diffeomorphic to $M$. The reason one wants such estimates is that they prevent noncompactness of sublevel sets, and therefore allow one to show local minima exist. To see this, we use another two theorems from differential geometry.

Theorem 9.8 (Gromov) For any $n$ there is a constant $c_{n}>0$ such that for any Riemannian metric $(M, g)$ if the Ricci curvature is greater than -1 (which occurs if the sectional curvature $|K| \leq 1$, as always assumed by Nabutovsky and Weinberger) then

$$
\operatorname{vol}(M, g) \geq c_{n}\|M\|,
$$

where $\|M\|$ denotes the simplicial norm of $(M, g)$.

Guaranteeing that $\left\|P_{k}\right\|>1$ will also guarantee by Theorem 9.8 that $\operatorname{vol}\left(M \# P_{k}\right)>c_{n}>0$, because $\left\|M \# P_{k}\right\|=\|M\|+\left\|P_{k}\right\|$, The final link in the chain is the next theorem.

Theorem 9.9 (Cheeger-Gromov) Let $T$ be the set of metrics $g$ on a manifold $M$ such that:

1. $|K| \leq 1 ; \quad$ (The sectional curvature of $(M, g)$ has absolute value $\leq 1$.)

2. $\operatorname{vol}(g)>\epsilon>0 ; \quad$ (The volume of $(M, g)$ is bounded away from 0.)

3. $\operatorname{diam}(g)<c<\infty$. (The diameter of $(M, g)$ is bounded below $\infty$.)

Then $T$ is compact, and hence the diameter function $D(g)$ has a minimum on this set $T$.

We have ignored issues of the analytic regularity of the metrics.

Now we can put it all together as follows. Fix a sublevel set $U=$ $f^{-1}((0, D(g)+y))$ for some point $g \in \operatorname{Met}(M)$. This restriction gives a bound on the diameter and yields (3) of Theorem 9.9. The universal assumption of Nabutovsky and Weinberger guarantees (1). Their construction in Theorem 9.7 guarantees that (2) can be assumed to hold for a class of components of the sublevel set that contain manifolds impossible to algorithmically disentangle from $M$.

As a result of (2), there must be infinitely many components of the sublevel sets of $D$ on $\operatorname{Met}(M)$ which satisfy the volume bound $v>c_{n} / 2$, for if not, one could use this to enumerate $T$ as follows. Keep a list of the finitely 
many components which satisfy the volume bound (by recording an element of each). Check whether $N_{k}$ lies in a component of the space of manifolds with $|K|<1$ and diam $<2 D$, that satisfies the volume bound. (This is algorithmically checkable.) If not, $k \in S$ (by Gromov's theorem). If so, check whether it is one of the finitely many exceptional components on our list. If so, then also $k \in S$, otherwise $k \in T$. Now, one has in $\operatorname{Met}(M)$ many components of sublevel set satisfying the volume inequality. The CheegerGromov theorem implies the compactness of each of these components. Since $D$ is a continuous function on these compact spaces, in each component it has a global minimum. The global minima are the desired local minima, since a local minimum for a function is exactly the same thing as a global minimum of the function restricted to an open set. The proof is complete.

\section{References}

[1] [Adjan, 1955]

S. I. Adjan, The algorithmic unsolvability of checking certain properties of groups, Dokl. Akad. Nauk SSSR 103 (1955) 533-535 (in Russian).

[2] [Adjan, 1958a]

On algorithmic problems in effectively complete classes of groups (Russian), Doklady Akad. Nauk SSSR, 123 (1958), 13-16.

[3] [Adjan, 1958b]

S. I. Adjan, On algorithmic problems in effectively complete classes of groups (Russian), Doklady Akad. Nauk SSSR, 123 (1958), 13-16.

[4] [Bochnak, Coste, and Roy]

J. Bochnak, M. Coste, and M. F. Roy, Geometrie Alg/'ebrique R/'eele, Springer, 1987.

[5] [Boone, 1954] W. W. Boone, Certain simple unsolvable problems of group theory, Indig. Math. 16 (1954), 231-237; 17 (1955), 252-256, 571-577; 19 (1957), 22-27, 227-232.

[6] [Boone, 1959]

W. W. Boone, The word problem, Annals of Math. 70 (1959), 207-265.

[7] [Boone, 1966]

W. W. Boone, Word problems and recursively enumerable degrees of unsolvability, A sequel on finitely presented groups, Annals of Math. 84 (1966) 49-84. 
[8] [Boone, Haken, Poenaru 1968]

W. W. Boone, W. Haken, and V. Poenaru, On recursively unsolvable problems in topology and their classification, In: Contributions to mathematical logic, H. Arnold Schmidt, K. Schütte, H. J. Thiele, eds., North-Holland, Amsterdam, 1968.

[9] [Britton, 1963]

J. L. Britton, The word problem, Ann. Math. 77 (1963) 16-32.

[10] [Cheeger, 1970]

J. Cheeger, Finiteness theorems for Riemannian manifolds, Amer. J. Math. 92 (1970), 61-74.

[11] [Chavel, 1993]

I. Chavel, Riemannian geometry-A modern introduction, Cambridge Univ. Press, Cambridge, 1993.

[12] [Church, 1936]

A. Church, An unsolvable problem of elementary number theory, American J. of Math., 58 (1936), 345-363.

[13] [Coste, 1982]

M. Coste, Ensembles semi-algebriques, in: Geometric Algebrique Reelle et formes quadratiques, Journees S.M.F. Universite de Rennes (J.-L. Colliot-Thelene, M. Coste, L. Mahe, M.-F. Roy eds., Springer Lecture Notes in Math. 959 (1982), 109-138.

[14] [Csima, 2003]

B. F. Csima, Computable model theory, Ph.D. thesis, The University of Chicago, 2003.

[15] [Csima-Soare, ta]

B. F. Csima and R. I. Soare, Applications of computability to differential geometry, to appear.

[16] [Davis, 1965]

M. Davis, The Undecidable, Raven Press, Hewlett, NY, 1965.

[17] [Davis, 1973]

M. Davis, Hilbert's tenth problem is unsolvable, Amer. Math. Monthly 80 (1973), 233-269.

[18] [Dieudonné, 1960]

Foundations of modern analysis, Academic Press, New York, 1960. 
[19] [Do Carmo, 1992]

M. DoCarmo, Riemannian Geometry, Birkhäuser, Boston, Basel, Berlin, 1992.

[20] [Freedman, 1982]

M. Freedman, The topology of four-dimensional manifolds, J. Differential Geometry 17 (1982), 357-453.

[21] [Gromov, 1981]

M. L. Gromov, Hyperbolic manifolds, groups and actions, in: Riemannian surfaces and related topics, I. Kra and B. Maskit, ed., Ann. of Math. Studies 97 (1981), 183-215.

[22] [Guillemin-Pollack, 1974]

V. Guillemin and A. Pollack, Differential Topology, Prentice-Hall, Englewood Cliffs, New Jersey, 1974.

[23] [Haken, 1973]

W. Haken, Connections between topological and group theoretical decision problems, In: Word problems: decision problems and the Burnside problem in group theory, Eds. W. W. Boone, F. B. Cannonito, and R. C. Lyndon, Studies in Logic and the Foundations of Mathematics, vol. 71, North-Holland, Amsterdam, London, 1973, 427-441.

[24] [Kervaire and Milnor, 1963]

M. Kervaire and J. Milnor, Groups of homotopy spheres I, Ann. Math., 77 (1963), 504-537.

[25] [Levine, 1985]

J. Levine, Lectures on groups of homotopy spheres, Lecture notes in Math 1126, Algebraic and geometric topology, 1985, Springer-Verlag, pp. $62-95$.

[26] [Massey, 1967]

W. S. Massey, Algebraic topology: An introduction, Harcourt, Brace \& World, Inc., New York, Chicago, 1967.

[27] [Nabutovsky, 1995a]

A. Nabutovsky, Einstein structures: Existence versus uniqueness, Geometric and Functional Analysis, 5, No. 1, (1995), 76-91.

[28] [Markov, 1951]

A. A. Markov, Impossibility of algorithms for recognizing some proper- 
ties of associative systems, Dokl. Akad. Nauk SSSR 77 (1951) 953-956

(in Russian).

[29] [Markov, 1958]

A. A. Markov, Insolubility of the problem of homeomorphy, In: Proc. Intern. Congress of Mathematicians, 1958, Cambridge University Press, Cambridge, 300-306.

[30] [Miller, 1971]

C. Miller, On group-theoretic decision problems and their classification, Annals of Math. Studies, No. 68, Princeton University Press, Princeton, New Jersey, 1971.

[31] [Miller, 1989]

C. Miller, Decision problems for groups - survey and reflections, in: Combinatorial group theory, eds. G. Baumslag, C. Miller, SpringerVerlag, Heidelberg, New York, 1989, 1-59.

[32] [Milnor, 1963]

J. Milnor, Morse Theory, Princeton University Press, Princeton, N.J., No. 51, 1963.

[33] [Milnor, 1965]

J. Milnor, Topology from a differential viewpoint, University of Virginia Press, 1965.

[34] [Milnor, 1965b]

J. Milnor, Lectures on the $h$-cobordism Theorem, Princeton Univ. Press, Princeton, New Jersey, 1965.

[35] [Nabutovsky, 1995a]

A. Nabutovsky, Einstein structures: Existence versus uniqueness, Geometric and Functional Analysis, 5, No. 1, (1995), 76-91.

[36] [Nabutovsky, 1995b]

A. Nabutovsky, Non-Recursive Functions, Knots with "Thick Ropes," and Self-Clenching "thick" Hypersurfaces, Comm. Pure Appl. Math. 48 (1995), 381-428.

[37] [Nabutovsky, 1996a] A. Nabutovsky, Disconnectedness of sublevel sets of some Riemannian functionals, Geometric and Functional Analysis, 6 (1996), 703-725. 
[38] [Nabutovsky, 1996b]

A. Nabutovsky, Geometry of the space of triangulations of a compact manifold, Communications in Mathematical Physics, 181 (1996), 303330 .

[39] [Nabutovsky, 1996c]

A. Nabutovsky, Fundamental group and contractible closed geodesics, Comm. on Pure and Appl. Math. 49 (1996), 1257-1270.

[40] [NW, 1996]

A. Nabutovsky and S. Weinberger, Algorithmic unsolvability of the triviality problem for multidimensional knots, Comment. Math. Helv. 71 (1996) 426-434.

[41] [NW, 1999]

A. Nabutovsky and S. Weinberger, Algorithmic aspects of homeomorphism problems, in: Rothenberg Festschrift, Contemp. Math. 231 (1999), 145-250.

[42] [NW1]

A. Nabutovsky and S. Weinberger, Variational problems for Riemannian functionals and arithmetic groups, Publications Mathématiques, Institut des Hautes Études Scientifiques, no. 92, (2000), 5-62.

[43] [NW2]

A. Nabutovsky and S. Weinberger, The Fractal Nature of Riem/Diff I, to appear in Geometrica Dedicata in 2003-2004.

[44] [NW3]

A. Nabutovsky and S. Weinberger, Critical points of Riemannian functionals and arithmetic groups, Math Publ d'IHES, 92 (2000) 5-62.

[45] [Novikov, 1955]

P. S. Novikov, On the algorithmic unsolvability of the word problem in groups (Russian), Trudy Mat. Inst. Steklov no. 44 Izdat Akad. Nauk SSSR, Moscow, 1955.

[46] [Petersen, 1993]

P. Petersen, Gromov-Hausdorff convergence of metric spaces, in: Differential Geometry: Riemannian Geometry, ed. by R. Greene, S.T. Yau, Proceedings of AMS Symposia in Pure Mathematics, 54:3 (1993), 489504. 
[47] [Petersen, 1998]

P. Petersen, Riemannian Geometry, Graduate Texts in Mathematics 171, Springer-Verlag, Heidelberg, New York, 1998.

[48] Poincaré [1895]

H. Poincaré, Analysis situs, J. de l'Êcole Poly., Paris (2) 1 (1895), $1-123$.

[49] [Rabin, 1958]

M. O. Rabin, Recursive unsolvability of group theoretic problems, Ann. of Math., 67 (1958), 172-194.

[50] [Rotman, 1995]

J. J. Rotman, An introduction to the theory of groups, Springer-Verlag, Heidelberg, New York, 1995.

[51] Reid [1970]

Hilbert, Springer-Verlag, New York, Heidelberg, 1970.

[52] [Sapir-Birget-Rips, 2002]

M. V. Sapir, J. C. Birget, E. Rips, Isoperimetric and isodiametric functions of groups, Annals of Math. 156 (2002), 467-518.

[53] [Smale, 1961]

S. Smale, Generalized Poincaré's conjecture in dimensions greater than four, Ann. Math. 74, (1961), 391-406.

[54] [Soare, 1987]

R. I. Soare, Recursively Enumerable Sets and Degrees: A Study of Computable Functions and Computably Generated Sets, Springer-Verlag, Heidelberg, 1987.

[55] [Spivak, 1965]

M. Spivak, Calculus on Manifolds, Benjamin Press, New York, 1965.

[56] [Spivak, 1979]

M. Spivak, A comprehensive introduction to differential geometry, vols.

I-V, Publish or Perish, Wilmington, 1979.

[57] J. W. Stallings, Polyhedral homotopy spheres, Bull. Amer. Math. Soc. 66 (1960), 485-488.

[58] [Turing, 1936]

A. M. Turing, On computable numbers, with an application to the 
Entscheidungsproblem, Proc. London Math. Soc. 42 (1936), 230-265;

A correction, ibid. 43 (1937), 544-546; reprinted in Davis [1965], 115154 .

[59] [Weinberger, ta]

S. Weinberger, Computers, Rigidity, and Moduli: Computation and the large scale geometry of moduli spaces, Princeton University Press, to appear in 2004. (Book from Porter Lectures given at Rice University.)

Department of Mathematics

UNIVERSity OF CHICAGO

Chicago, Illinois 60637-1546

E-mail:ssoare@math.uchicago.edu or soare@cs.uchicago.edu

Anonymous ftp: cs.uchicago.edu: ftp/pub/users/soare

World Wide Web: http://www.cs.uchicago.edu/ soare 